\title{
Scattering of Two Plane Electromagnetic Waves in the Non-Linear Born-Infeld Electrodynamics
}

\author{
B. M. Barbashov and N. A. Chernikov \\ Joint Institute for Nuclear Research \\ Laboratory of Theoretical Physics \\ Dubna
}

Received May 15, 1966

\begin{abstract}
The problem of scattering of two plane waves is exactly solved in the Born-Infeld electrodynamics. The shape and the direction of the plane waves after scattering turn out to be unchanged. There is only a change in the wave phase by the value which is the product of its frequency and the momentum of the incident wave. It is shown that in the interaction region the solution becomes a multiplevalued function of $x, t$ when the electromagnetic field exceeds a certain value which plays the role of an absolute field scale.
\end{abstract}

\section{Introduction}

In the authors' paper [1] the identity was established between the equation

$$
\left(1-\varphi_{t}^{2}\right) \varphi_{x x}+2 \varphi_{x} \varphi_{t} \varphi_{x t}-\left(1+\varphi_{x}^{2}\right) \varphi_{t t}=0
$$

describing the non-linear scalar field in the two-dimensional pseudoEuclidean space $x, t$ with the Born-Infeld Lagrangian [2]

$$
\mathscr{L}=1-\sqrt{1+\varphi_{x}^{2}-\varphi_{t}^{2}}
$$

and the equation of the minimal surfaces $z=\varphi(x, t)$ in the threedimensional pseudo-Euclidean space with the metric $d s^{2}=d t^{2}-d x^{2}-d z^{2}$ where the surface area is given by the integral

$$
S=-\iint\left(\sqrt{1+\varphi_{x}^{2}-\varphi_{t}^{2}}-1\right) d x d t .
$$

The extremum conditions of this integral lead also to eqs. (1). The solution of the Cauchy problem for eq. (1) was found there [1].

On the basis of the analogy between eq. (1) and the minimal surfaces a generalization of the Lagrangian (2) was suggested to the case of many non-linear fields interacting in a definite way in the two-dimensional space. This system gives also the exact solution. To make this generalization we consider the problem of the minimal two-dimensional surface in a $n+2$-dimensional space with the metric $d s^{2}=d t^{2}-d x^{2}-\sum_{i=1}^{n} d z_{i}^{2}$. 
Let the surface be given by the equations

$$
z_{1}=\varphi_{1}(x, t) ; z_{2}=\varphi_{2}(x, t) ; \ldots z_{n}=\varphi_{n}(x, t) .
$$

Then the surface area is determined by the integral

$$
S=-\iint\left(\sqrt{\left(1+\sum_{i=1}^{n} \phi_{i x}^{2}\right)\left(1-\sum_{i=1}^{n} \phi_{i t}^{2}\right)+\left(\sum_{i=1}^{n} \phi_{i x} \varphi_{i t}\right)^{2}}-1\right) d x d t .
$$

The quantity $S$ may be interpreted as the action function of the $n$ field system with the Lagrangian density

$$
\mathscr{L}=1-\sqrt{\left(1+\sum_{i=1}^{n} \phi_{i, x}^{2}\right)\left(1-\sum_{i=1}^{n} \phi_{i t}^{2}\right)+\left(\sum_{i=1}^{n} \varphi_{i x} \varphi_{i t}\right)^{2}}
$$

We write the equations of motion for this system

$$
\begin{array}{r}
\left(1-\sum_{i=1}^{n} \varphi_{i t}^{2}\right) \varphi_{j x, x}+2\left(\sum_{i=1}^{n} \varphi_{i x} \varphi_{i t}\right) \varphi_{j x, t}-\left(1+\sum_{i=1}^{n} \varphi_{i, x}^{2}\right) \varphi_{j t, t}=0 . \\
(j=1,2, \ldots, n)
\end{array}
$$

In paper [1] the Cauchy problem for eq. (7) was solved for an arbitrary number $n$ of fields. For Cauchy data

$$
\left.\varphi_{i}(x, t)\right|_{t=0}=a_{i}(x) ;\left.\varphi_{i t}(x, t)\right|_{t=0}=b_{i}(x)
$$

the solution is obtained in a parametric form

$$
\begin{gathered}
t(\alpha, \beta)=\frac{\beta-\alpha}{2}+\frac{1}{2} \int_{\alpha}^{\beta} \mathscr{H}(\lambda) d \lambda \\
x(\alpha, \beta)=\frac{\beta+\alpha}{2}+\frac{1}{2} \int_{\alpha}^{\beta} \mathscr{G}(\lambda) d \lambda \\
\varphi_{i}(\alpha, \beta)=\frac{a_{i}(\alpha)+a_{i}(\beta)}{2}+\frac{1}{2} \int_{\alpha}^{\beta} \Pi_{i}(\lambda) d \lambda .
\end{gathered}
$$

The quantities $\Pi_{i}(x), \mathscr{G}(x), \mathscr{H}(x)$ in eqs. (9) have an important physical meaning. $\Pi_{i}(x)$ is a canonical momentum of the field $\varphi_{i}(x, t)$ at $t=0$.

$$
\begin{gathered}
\Pi_{i}(x)=\left.\cdot \frac{\partial \mathscr{L}}{\partial \varphi_{i, 6}}\right|_{t=0}= \\
=\frac{b_{i}(x)\left[1+\sum_{i=1}^{n} a_{i}^{\prime 2}(x)-a_{i}^{\prime}(x) \sum_{i=1}^{n} a_{i}^{\prime}(x) b_{i}(x)\right]}{\sqrt{\left(1+\sum_{i=1}^{n} a_{i}^{\prime 2}(x)\right)\left(1-\sum_{i=1}^{n} b_{i}^{2}(x)\right)+\left(\sum_{i=1}^{n} a_{i}^{\prime} b_{i}\right)^{2}}}
\end{gathered}
$$


$\mathscr{G}(x)$ is the momentum density of the field system with the Lagrangian (6) at $t=0$.

$$
\begin{gathered}
\mathscr{G}(x)=-\left.\sum_{i=1}^{n} \frac{\partial \mathscr{L}}{\partial \varphi_{i t}} \varphi_{i x}\right|_{t=0}=-\sum_{i=1}^{n} \Pi_{i}(x) a_{i}^{\prime}(x)= \\
-\frac{-\sum_{i=1}^{n} a_{i}^{\prime}(x) b_{i}(x)}{\sqrt{\left(1+\sum_{i=1}^{n} a_{i}^{\prime 2}(x)\right)\left(1-\sum_{i=1}^{n} b_{i}^{2}(x)\right)+\left(\sum_{i=1}^{n} a_{i}^{\prime}(x) b_{i}^{\prime}(x)\right)^{2}}}
\end{gathered}
$$

Finally, $\mathscr{H}(x)$ is the Hamiltonian density of the system with the Lagrangian $(6)$

$$
\begin{aligned}
\mathscr{H}(x) & =\left(\sum_{i=1}^{n} \Pi_{i}(x t) \varphi_{i, t}(x t)-\mathscr{L}\right)_{t=0}=\frac{1+\sum_{i=1}^{n}+a_{i}^{\prime 2}(x)}{1-\mathscr{L}(x, 0)}-1= \\
& =\sqrt{1+\sum_{i=1}^{n}\left(a_{i}^{\prime 2}(x)+\Pi_{i}^{2}(x)\right)+\left(\sum_{i=1}^{n} a_{i}^{\prime}(x) \Pi_{i}(x)\right)^{2}}-1 .
\end{aligned}
$$

We show that the problem of scattering of two plane waves in the Born-Infeld electrodynamics reduces to the solution of eqs. (7) for $n=2$.

\section{Solution for the scattering problem}

We consider the electromagnetic field equations in the Born-Infeld electrodynamics [2]. The Lagrangian of this system is of the form

where

$$
\mathscr{L}=1-\sqrt{1+F-G^{2}}
$$

$$
F=\frac{1}{2} f_{i k} f^{k i}, \quad G=\frac{1}{4} \varepsilon^{s k l m} f_{s k} f_{l m}
$$

$\varepsilon^{s k l m}$ is a completely antisymmetric tensor $\varepsilon^{1234}=\frac{1}{2} ; f_{i k}$ are the components of the electromagnetic field tensor.

If the vector-potential of the electromagnetic field is introduced then $f_{i k}=\frac{\partial \varphi_{k}}{\partial x_{i}}-\frac{\partial \varphi_{i}}{\partial x_{k}} ; \varphi_{i}$ obeys the Lorentz condition $\frac{\partial \varphi^{k}}{\partial x_{k}}=0$. In varying with respect to $\varphi_{i}$, from eq. (13) follow the equation for the electromagnetic field:

$$
\sum_{i=1}^{n} \frac{\partial}{\partial x^{i}}\left(\frac{\partial \mathscr{L}}{\partial \frac{\partial \varphi_{k}}{\partial x^{i}}}\right)=0 \quad(k=1,2,3,4)
$$

or

$$
\sum_{i=1}^{4} \frac{\partial}{\partial x^{i}} p^{i k}=0, \quad \text { where } \quad p^{i k}=\frac{f^{i k}-G \varepsilon^{i k e m} f_{e m}}{\sqrt{1+F-G^{2}}} .
$$

It is easy to prove that the plane wave of an arbitrary shape $\varphi_{i}(x, y, z, t)=\varphi_{i}((\mathbf{k} \mathbf{r})-|\mathbf{k}| t)$ is the solution for eqs. (14). Indeed, for $22^{*}$ 
the plane wave with the four-momentum eqs. (14) takes on the form:

$$
\sum_{i=1}^{4} k_{i} \frac{\partial p^{i l}}{\partial s}=0 ;(l=1,2,3,4), \quad \text { where } s=(\mathbf{k r})-|\mathbf{k}| t .
$$

The Lorentz condition is then written in the form

$$
\sum_{i=1}^{4} k_{i} \frac{\partial \varphi^{i}(s)}{\partial s}=0, \quad k^{2}=0 .
$$

Further it is easy to see that for the plane wave, taking into account conditions $(14 b)$, on has the equalities $F=G=0$. Thus $p^{i l}=f^{i l}$ as in the case of the linear Maxwell equations. The equation (14a), taking into account $(14 b)$, is then satisfied by

$$
\sum_{i=1}^{4} k_{i} \frac{\partial}{\partial s}\left(k^{i} \frac{\partial \varphi^{l}}{\partial s}-k^{l} \frac{\partial \varphi^{i}}{\partial s}\right)=0 .
$$

The sum of the two plane waves

$$
\varphi_{i}(x, y, z, t)=\varphi_{1 i}\left(k_{1}^{l} x_{l}\right)+\varphi_{2 i}\left(k_{2}^{l} x_{l}\right)
$$

satisfies also eqs. (14) in a space-time region where they are not overlapped, i.e. in the region where one of the functions is zero.

We may assume that these waves are moving along the axis $x$. This can be always obtained by the Lorentz transformation. In fact, let us direct the time axis along the four-vector $\mathbf{k}_{1}+\mathbf{k}_{\mathbf{2}}$ and the $x$-axis along $\mathbf{k}_{1}-\mathbf{k}_{2}$, then in a new frame of reference

$$
\begin{gathered}
\left(\mathbf{k}_{1} \mathbf{r}\right)-\left|\mathbf{k}_{1}\right| t=\sqrt{\frac{\left(\mathbf{k}, \mathbf{k}_{2}\right)}{2}}\left(x^{\prime}-t^{\prime}\right) \\
\left(\mathbf{k}_{2} \mathbf{r}\right)-\left|\mathbf{k}_{2}\right| t=-\sqrt{\frac{\left(\mathbf{k}_{1} \mathbf{k}_{2}\right)}{2}}\left(x^{\prime}+t^{\prime}\right) .
\end{gathered}
$$

Since eqs. (14) and the Lagrangian (13) are invariant under the Lorentz transformation we are able to formulate our problem in the following way.

We find the solution for eqs. (14) satisfying the following asymptotic conditions, as $t \rightarrow-\infty$ the solution must turn into two plane waves moving in opposition directions:

$$
\lim _{u \rightarrow \infty} \varphi_{i}(x, y, z, t)=\psi_{2, i}(v) ; \lim _{v \rightarrow-\infty} \varphi_{i}(x, y, z t)=\psi_{1 i}(u)
$$

where $u=x-t, v=x+t$ are the isotropic coordinates. The limiting values of the vector-potential must also obey the Lorentz conditior which is expressed in variables $u, v$ as follows:

$$
\begin{aligned}
& \frac{\partial \psi_{4,1}(u)}{\partial u}+\frac{\partial \psi_{1,1}(v)}{\partial v}=0 \\
& \frac{\partial \psi_{4,2}(v)}{\partial v}-\frac{\partial \psi_{1,2}(v)}{\partial v}=0 .
\end{aligned}
$$


To satisfy the conditions (15) we shall find a solution for eqs. (14) which would be independent of $y$ and $z$, i.e. we assume that $\varphi_{i}$ are functions of the two variables $x, t$ only. In this case eqs. (14) takes on the form:

$$
\begin{aligned}
& \frac{\partial}{\partial t}\left(\frac{\left.\frac{\partial \varphi_{4}}{\partial x}-\frac{\partial \varphi_{1}}{\partial t}\right)}{1-\mathscr{L}}\right)=0 ; \frac{\partial}{\partial x}\left(\frac{\frac{\partial \varphi_{1}}{\partial t}-\frac{\partial \varphi_{4}}{\partial x}}{1-\mathscr{L}}\right)=0, \\
& \frac{\partial}{\partial t}\left(\frac{-\frac{\partial \varphi_{2}}{\partial t}+\frac{\partial \varphi_{3}}{\partial x}\left(\frac{\partial \varphi_{2}}{\partial x} \frac{\partial \varphi_{3}}{\partial t}-\frac{\partial \varphi_{2}}{\partial t} \frac{\partial \varphi_{3}}{\partial x}\right)}{1-\mathscr{L}}\right)+ \\
& +\frac{\partial}{\partial x}\left(\frac{\frac{\partial \varphi_{3}}{\partial x}-\frac{\partial \varphi_{2}}{\partial t}\left(\frac{\partial \varphi_{2}}{\partial t} \frac{\partial \varphi_{3}}{\partial x}-\frac{\partial \varphi_{2}}{\partial x} \frac{\partial \varphi_{3}}{\partial t}\right)}{1-\mathscr{L}}\right)=0, \\
& \frac{\partial}{\partial t}\left(\frac{-\frac{\partial \varphi_{3}}{\partial t}+\frac{\partial \varphi_{2}}{\partial x}\left(\frac{\partial \varphi_{2}}{\partial t} \frac{\partial \varphi_{3}}{\partial x}-\frac{\partial \varphi_{2}}{\partial x} \frac{\partial \varphi_{3}}{\partial t}\right)}{1-\mathscr{L}}\right)+ \\
& +\frac{\partial}{\partial x}\left(\frac{\frac{\partial \varphi_{3}}{\partial x}-\frac{\partial \varphi_{2}}{\partial t}\left(\frac{\partial \varphi_{2}}{\partial t} \frac{\partial \varphi_{3}}{\partial x}-\frac{\partial \varphi_{2}}{\partial x} \frac{\partial \varphi_{3}}{\partial t}\right)}{1-\mathscr{L}}\right)=0 .
\end{aligned}
$$

From the first two equations (16) it follows immediately that

$$
\frac{\partial \varphi_{4}}{\partial x}-\frac{\partial \varphi_{1}}{\partial t}=c(1-\mathscr{L}), \text { where } \quad c=\text { const . }
$$

When $\varphi_{i}$ depends on $x, t$ the Lorentz condition relates only $\varphi_{1}$ and $\varphi_{2}$

$$
\frac{\partial \varphi_{4}}{\partial t}-\frac{\partial \varphi_{1}}{\partial x}=0
$$

Summing up and subtracting (17) and (17a) and going over to the isotropic variables $u, v$ we get

$$
\frac{\partial \varphi_{4}}{\partial u}+\frac{\partial \varphi_{1}}{\partial u}=c(1-\mathscr{L}) ; \quad \frac{\partial \varphi_{4}}{\partial v}-\frac{\partial \varphi_{1}}{\partial v}=c(1-\mathscr{L}) .
$$

Now comparing eqs. (18) with (15a), the latter being obtained from (15a) at $u \rightarrow-\infty$ and $v \rightarrow \infty$ according to the limiting conditions (15), we see that to obtain agreement with the limiting values it is necessary to put in eq. (17) $c=0$. From the physical point of view this means the following. The quantity $\left(\frac{\partial \varphi_{4}}{\partial x}-\frac{\partial \varphi_{1}}{\partial t}\right)(1-\mathscr{L})^{-1}$ in the Born-Infeld theory is an $x$-component of the electrical induction vector $D$. According to (17) it is constant. If $c \neq 0$ then besides the plane wave, at infinity there exists a constant longitudinal electric induction, as there would exist a condenser with plates separated to infinity.

When $c=0$ the Lagrangian (13) takes on the same form as (6) for the two fields since when the field depends only on $x$ and $t$ the components $\varphi_{4}$ and $\varphi_{1}$ enter $\mathscr{L}$ as $\frac{\partial \varphi_{4}}{\partial x}-\frac{\partial \varphi_{1}}{\partial t}$ and consequently, due to (17) 
cancel each other in $\mathscr{L}^{*}$

$$
1-\mathscr{L}=\sqrt{\left(1+\sum_{i=2}^{3} \phi_{i x}^{2}\right)\left(1-\sum_{i=2}^{3} p_{i t}^{2}\right)+\left(\sum_{i=2}^{3} \phi_{i x} \varphi_{i t}\right)^{2}}
$$

The last two equations of (16) for $\varphi_{2}$ and $\varphi_{3}$ coincide with the corresponding equations (7) for $j=2,3$.

Thus, our problem is reduced to the solution of eq. (7) with asymptotic conditions (15).

Solving the Cauchy problem (9) for the initial data (8) we can obtain the desired solution satisfying (15).

With this aim we investigate the asymptotic behaviour of the solution (9), letting $v$ tend to minus infinity for a fixed $u$, and $u$ to infinity for a fixed $v . u$ and $v$ are the isotropic coordinates (15); in both cases $t=-\frac{v-u}{2} \rightarrow-\infty$. Further we assume that $a_{i}(x)$ and $b_{i}(x)$ rather fast decrease at infinity.

The parameters $\alpha$ and $\beta$ in (9) are suitable for the Cauchy problem while the parameters $\mu=\mu(\alpha)$ and $\nu=\nu(\beta)$ are more convenient for the solution of the problem of plane wave scattering (15). We are led to the latter parameters by considering the limits of eqs. (9) for $\beta \rightarrow-\infty$ and for $\alpha \rightarrow \infty$. In the first case from (9) we get

$$
\begin{gathered}
\lim _{\beta \rightarrow-\infty} u=\lim _{\beta \rightarrow-\infty}(x-t)=\alpha+\frac{1}{2} \int_{-\infty}^{\alpha}[\mathscr{H}(\lambda)-\mathscr{G}(\lambda)] d \lambda=\mu(\alpha) \\
\lim _{\beta \rightarrow-\infty} v=\lim _{\beta \rightarrow-\infty}(x+t)=-\infty \\
\lim _{\beta \rightarrow-\infty} \varphi_{i}(\alpha, \beta)=\frac{a_{i}(\alpha)}{2}-\frac{1}{2} \int_{-\infty}^{\alpha} \Pi_{i}(\lambda) d \lambda=\psi_{i 1}(\mu) .
\end{gathered}
$$

In the second one

$$
\begin{gathered}
\lim _{\alpha \rightarrow \infty} u=\lim _{\alpha \rightarrow \infty}(x-t)=\infty \\
\lim _{\alpha \rightarrow \infty} v=\lim _{\alpha \rightarrow \infty}(x+t)=\beta-\frac{1}{2} \int_{\beta}^{\infty}[\mathscr{H}(\lambda)+\mathscr{G}(\lambda)] d \lambda=v(\beta) \\
\lim _{\alpha \rightarrow \infty} \varphi_{i}(\alpha, \beta)=\frac{a_{i}(\beta)}{2}-\frac{1}{2} \int_{\beta}^{\infty} \Pi_{i}(\lambda) d \lambda=\psi_{i 2}(v) .
\end{gathered}
$$

Below it will be shown that the introduced functions $\psi_{i, 1}$ and $\psi_{i, 2}$ coincide with the limiting values of (15).

* When $c \neq 0 \varphi_{1}$ and $\varphi_{2}$ can be also excluded from $\mathscr{L}$ and an exact solution can be obtained. In this case

$$
1-\mathscr{L}=\frac{1}{\sqrt{1+c^{2}}} \sqrt{\left(1+\sum_{i=2}^{3} \phi_{i, x}^{2}\right)\left(1-\sum_{i=2}^{3} \phi_{i t}^{2}\right)+\left(\sum_{i=2}^{3} \phi_{i x} \varphi_{i t}\right)^{2}} .
$$


This follows from the fact that $\mu=\mu(\alpha)$ and $\nu=\nu(\beta)$ are monotoneously increasing functions since $\mathscr{H}(\lambda)>\mathscr{G}(\lambda)$. Consequently, the transformations from $\alpha, \beta$ to $\mu, \nu$ are one-to-one, and as we saw from eqs. (20) and (21) when $\beta \rightarrow-\infty$, then $v \rightarrow-\infty$ and when $\alpha \rightarrow \infty$, then $u \rightarrow \infty$. From (20) (21) and (9) it follows that

$$
\varphi_{i}(\alpha, \beta)=\psi_{i, 1}(\mu)+\psi_{i 2}(\nu)+\frac{1}{2} \int_{-\infty}^{\infty} \Pi_{i}(\lambda) d \lambda .
$$

The quantity $\frac{1}{2} \int_{-\infty}^{\infty} \Pi_{i}(\lambda) d \lambda$ can be expressed in terms of $\psi_{i 1}$ and $\psi_{i 2}$ since from (20) and (21) we have

$$
\begin{gathered}
\lim _{\nu \rightarrow \infty} \psi_{i 2}(v)=0 ; \lim _{\mu \rightarrow-\infty} \psi_{i 1}(\mu)=0 \\
\lim _{\nu \rightarrow-\infty} \psi_{i 2}(\nu)=\lim _{\mu \rightarrow \infty} \psi_{i 1}(\mu)=\psi_{i 0}=-\frac{1}{2} \int_{-\infty}^{\infty} \Pi_{i}(\lambda) d \lambda .
\end{gathered}
$$

Here it is taken into account that

$$
\lim _{\alpha \rightarrow \pm \infty} \mu(\alpha)= \pm \infty, \lim _{\beta \rightarrow \pm \infty} v(\beta)= \pm \infty .
$$

Thus we can write

$$
\varphi_{i}(\mu, v)=\psi_{i 1}(\mu)+\psi_{i 2}(v)-\psi_{i 0} .
$$

Now we have only to express $u$ and $v$ as functions of $\mu, v$. By determining the functions $\mu(\alpha)$ and $v(\beta)$ in (20) and (21) and the expressions for $u$ and $v$ following from (9).

$$
\begin{aligned}
& u(\alpha, \beta)=x-t=\alpha+\frac{1}{2} \int_{\alpha}^{\beta}[\mathscr{G}(\lambda)-\mathscr{H}(\lambda)] d \lambda \\
& v(\alpha, \beta)=x+t=\beta+\frac{1}{2} \int_{\alpha}^{\beta}[\mathscr{H}(\lambda)+\mathscr{G}(\lambda)] d \lambda
\end{aligned}
$$

it may be concluded that

$$
\begin{gathered}
u=\mu(\alpha)+\frac{1}{2} \int_{-\infty}^{\beta}[\mathscr{G}(\lambda)-\mathscr{H}(\lambda)] d \lambda \\
v=v(\beta)+\frac{1}{2} \int_{\alpha}^{\beta}[\mathscr{G}(\lambda)+\mathscr{H}(\lambda)] d \lambda .
\end{gathered}
$$

In the first of these integrals we replace the variables $\sigma=v(\lambda)$ and in the second one $\sigma=\mu(\lambda)$. Since according to (20) and (21)

$$
\frac{d \mu}{d \lambda}=1+\frac{\mathscr{H}(\lambda)-\mathscr{G}(\lambda)}{2} ; \frac{d v}{d \lambda}=1+\frac{\mathscr{H}(\lambda)+\mathscr{G}(\lambda)}{2}
$$


then

$$
\begin{aligned}
& u=\mu(\alpha)-\int_{-\infty}^{v(\beta)} \frac{\mathscr{H}(\lambda)-\mathscr{G}(\lambda)}{2+\mathscr{H}(\lambda)+\mathscr{G}(\lambda)} d \sigma ; \quad \sigma=v(\lambda) \\
& v=v(\beta)+\int_{\mu(\alpha)}^{\infty} \frac{\mathscr{H}(\lambda)+\mathscr{G}(\lambda)}{2+\mathscr{H}(\lambda)-\mathscr{G}(\lambda)} d \sigma ; \quad \sigma=\mu(\lambda) .
\end{aligned}
$$

To express the integrands in (26) in terms of $\psi_{i 1}$ and $\psi_{i 2}$ we make use of $\psi_{i 1}$ and $\psi_{i 2}$ determined in (20) and (21) and find

$$
\begin{array}{ll}
\frac{d \psi_{i 1}(\sigma)}{d \sigma}=\frac{a_{i}^{\prime}(\lambda)-\Pi_{i}(\lambda)}{2+\mathscr{H}(\lambda)-\mathscr{G}(\lambda)}, & \sigma=\mu(\lambda) \\
\frac{d \psi_{i 2}(\sigma)}{d \sigma}=\frac{a_{i}^{\prime}(\lambda)+\Pi_{i}(\lambda)}{2+\mathscr{H}(\lambda)+\mathscr{G}(\lambda)}, & \sigma=v(\lambda) .
\end{array}
$$

Taking into account $\mathscr{G}(\lambda)$ and $\mathscr{H}(\lambda)$ determined in (11) and (12) it is easy to find that

$$
\begin{aligned}
\sum_{i=2}^{3}\left(\frac{d \psi_{i 1}(\sigma)}{d \sigma}\right)^{2} & =\frac{\mathscr{H}(\lambda)+\mathscr{G}(\lambda)}{2+\mathscr{H}(\lambda)-\mathscr{G}(\lambda)} \\
\sum_{i=2}^{3}\left(\frac{d \psi_{i 2}}{d \sigma}\right)^{2} & =\frac{\mathscr{H}(\lambda)-\mathscr{G}(\lambda)}{2+\mathscr{H}(\lambda)+\mathscr{G}(\lambda)} .
\end{aligned}
$$

Thus, we obtain

$$
\begin{aligned}
& u=\mu(\alpha)-\int_{-\infty}^{\nu(\beta)} \sum_{i=2}^{3} \psi_{i 2}^{\prime 2}(\sigma) d \sigma \\
& v=v(\beta)+\int_{\mu(\alpha)}^{\infty} \sum_{i=2}^{3} \psi_{i 1}^{\prime 2}(\sigma) d \sigma .
\end{aligned}
$$

The final solution expressed in new variables $\mu, v$ and initial functions $\psi_{i 1}, \psi_{i 2}$ is represented in the form

$$
\begin{aligned}
\varphi_{i}(\mu, v) & =\psi_{i, 1}(\mu)+\psi_{i, 2}(\nu)-\psi_{i, 0} \quad(i=2,3) \\
u & =\mu-\int_{-\infty}^{v} \sum_{i=2}^{3} \psi_{i, 2}^{\prime 2}(\sigma) d \sigma \\
v & =v+\int_{\mu}^{\infty} \sum_{i=2}^{3} \psi_{i, 1}^{\prime 2}(\sigma) d \sigma
\end{aligned}
$$

It is not difficult to prove that the solution satifies the limiting conditions (15) and eqs. (16). In fact, if the gauge is chosen so that $\varphi_{4}=0$ then from (17) with $c=0$ and from the Lorentz condition (17a) we have that $\varphi_{1}=$ const. This constant may be put to be equal to zero then only $\varphi_{2}(x, t)$ and $\varphi_{3}(x, t)$ remain different from zero. For then the solution is given in a parametric form (32). A direct substitution shows that it satisfies eqs. (16). From (32) for $\varphi_{2}$ and $\varphi_{3}$ we have also when $u \rightarrow \infty$ then $\mu \rightarrow \infty$, and $\nu$ becomes equal to $v$. 
Hence,

$$
\lim _{u \rightarrow \infty} \varphi_{i}=\psi_{i 2}(v) \quad(i=2,3) .
$$

If $v \rightarrow-\infty$ then $v \rightarrow-\infty$ as well, and $\mu$ becomes equal to $u$.

Consequently,

$$
\lim _{v \rightarrow-\infty} \varphi_{i}=\psi_{i 1}(u) \text {. }
$$

Thus, the problem of scattering of two plane waves in the Born-Infeld electrodynamics is solved.

Let us show how transforms our solution at $u \rightarrow-\infty$ and at $v \rightarrow \infty$. In both cases $t=\frac{v-u}{2} \rightarrow \infty$. It is obvious from (32) that when $u \rightarrow-\infty$ then $\mu \rightarrow-\infty$, and $v$ becomes equal to $v-H_{1}$ where

Consequently,

$$
H_{1}=\int_{-\infty}^{\infty} \sum_{i=2}^{3} \psi_{i 1}^{\prime 2}(\sigma) d \sigma \text {. }
$$

$$
\lim _{u \rightarrow-\infty} \phi_{i}=\psi_{i 2}\left(v-H_{1}\right)-\psi_{i 2}(-\infty) .
$$

When $v \rightarrow \infty$ then $v \rightarrow \infty$ and $\mu=u+H_{2}$ where

Hence

$$
H_{2}=\int_{-\infty}^{\infty} \sum_{i=2}^{3} \psi_{i, 2}^{\prime 2}(\sigma) d \sigma
$$

$$
\lim _{v \rightarrow \infty} \varphi_{i}=\psi_{i 1}\left(u+H_{2}\right)-\psi_{i 1}(\infty) .
$$

Thus, two plane electromagnetic waves after collision pass to two plane waves of the same shape but changed arguments. The quantities $H_{1}$, $-H_{2}$ which characterise the change of the arguments are equal to the momenta of the first and the second wave respectively. If desired, the collision may be considered in the center-of-mass system, where $H_{1}=-H_{2}$.

\section{Diseussion of the results}

The obtained solution (32) is, generally speaking, a multiple-valued function of $u, v$. Indeed, let us consider the dependence of $u, v$ on $\mu, v$.

where

$$
u=\mu-B(v) ; \quad v=v+A(\mu)
$$

$$
\left.B(v)=\int_{-\infty}^{\nu} \sum_{i=2}^{3} \psi_{i 2}^{\prime 2}(\sigma) d \sigma ; \quad A(\mu)=\int_{\mu}^{\infty} \sum_{i=2}^{3} \psi_{i 1}^{\prime 2}(\sigma) d \sigma\right)
$$

Excluding $v$ from (39) we get the equation

$$
\mu=u+B(v-A(\mu))
$$

defining $\mu$ as a function of $u, v$. The right-hand side of this equation is a monotonely increasing function of $\mu$ since its derivative is

$$
\sum_{i=2}^{3} \psi_{i 2}^{\prime 2}(v-A(\mu)) \sum_{j=2}^{3} \psi_{i 1}^{\prime 2}(\mu) \geqq 0 .
$$


322 BARBASHov and Chernikov: Scattering of Two Plane Electromagnetic Waves

Since

then

$$
0 \leqq A(\mu) \leqq H_{1}
$$

$$
u+B(v-A(\mu)) \leqq u+B(v) \leqq u+B\left(v+H_{1}\right)
$$

Consequently, all the roots of eq. (40) lie within the limits (42) and their number is always odd.

If at all the points

$$
\sum_{i=2}^{3} \psi_{i 1}^{\prime 2}(\mu) \sum_{j=2}^{3} \psi_{i 2}^{\prime 2}(v)<1
$$

then there is only one root of eq. (40) and the solution (32) is singlevalued function of $u, v$.

We can write an approximate expression for our solution as a function of $u, v$

$$
\varphi_{i} \cong \psi_{i 1}(u+B(v))+\psi_{i 2}(v-A(u))-\psi_{i 0} .
$$

The smaller is the expression

$$
\sum_{i=2}^{3} \psi_{i 1}^{\prime 2}(\mu) \sum_{j=2}^{3} \psi_{i 2}^{\prime 2}(v)
$$

the more accurate is the equality (44).

When the condition (43) is violated (in our units 1 plays the role of the absolute scale of the field gradient) the Jacobian

$$
\frac{\partial(u, v)}{\partial(\mu, v)}=1-\sum_{i=2}^{3} \psi_{i 1}^{\prime 2}(\mu) \sum_{j=2}^{3} \psi_{i}^{\prime 2}(\nu)
$$

is not everywhere different from zero. If the Jacobian (45) is zero then at these points the intensities of the field are infinite since

$$
\begin{aligned}
& E_{x}=0 \\
& E_{y}=\frac{\psi_{21}^{\prime}(\mu)\left[1-\sum_{i=2}^{3} \psi_{i 2}^{\prime 2}(v)\right]-\psi_{22}^{\prime}(v)\left[1-\sum_{i=2}^{3} \psi_{i 1}^{\prime 2}(\mu)\right]}{1-\sum_{i=2}^{3} \psi_{i 1}^{\prime 2}(\mu) \sum_{j=2}^{3} \psi_{j, 2}^{\prime 2}(v)} \\
& E_{Z}=0 .
\end{aligned}
$$

Now we may conclude that this theory, as it was assumed by its authors [2], loses the meaning when the intensities of the field become larger than some constant value (in our case 1) playing the role of the absolute field scale. As was already noted, if the initial data are chosen such that (43) is fulfilled there arise no ambiguities for weak fields; the theory turns into the Maxwell electrodynamics.

In conclusion the authors are grateful to D. I. BLokHINTzev for useful discussions of the problems concerned.

\section{References}

[1] Barbashov, B., et N. Chernikov: JETP 5, 50, 1296-1308 (1965).

[2] Born, M., and L. Infeld : Proc. Roy. Soc. A 144, 425 (1934). 